\title{
An Advanced Partial Discharge Recognition Strategy of Power Cable
}

\author{
Xiaotian Bi, Ang Ren, Simeng Li, Mingming Han, and Qingquan Li \\ Shandong Provincial Key Laboratory of UHV Transmission Technology \& Equipment, \\ School of Electrical Engineering, Shandong University, Jinan 250061, China \\ Correspondence should be addressed to Qingquan Li; lqq@sdu.edu.cn \\ Received 17 April 2015; Revised 16 July 2015; Accepted 29 July 2015 \\ Academic Editor: John N. Sahalos \\ Copyright (c) 2015 Xiaotian Bi et al. This is an open access article distributed under the Creative Commons Attribution License, \\ which permits unrestricted use, distribution, and reproduction in any medium, provided the original work is properly cited. \\ Detection and localization of partial discharge are very important in condition monitoring of power cables, so it is necessary to \\ build an accurate recognizer to recognize the discharge types. In this paper, firstly, a power cable model based on FDTD simulation \\ is built to get the typical discharge signals as training samples. Secondly, because the extraction of discharge signal features is crucial, \\ fractal characteristics of the training samples are extracted and inputted into the recognizer. To make the results more accurate, \\ multi-SVM recognizer made up of six Support Vector Machines (SVM) is proposed in this paper. The result of the multi-SVM \\ recognizer is determined by the vote of the six SVM. Finally, the BP neural networks and ELM are compared with multi-SVM. The \\ accuracy comparison shows that the multi-SVM recognizer has the best accuracy and stability, and it can recognize the discharge \\ type efficiently.
}

\section{Introduction}

With the development of the power industry and urbanization in China, the power cable is used everywhere. As a result, the detection and localization of partial discharge are becoming more and more important in condition monitoring of power cables. Building an accurate model of cables helps analyzing the propagation characteristics of electromagnetic pulse and recognizing its type accurately when partial discharge happens in the cable. So, accurate model is necessary $[1,2]$.

In recognizing the types of partial discharge, the extraction of discharge signal features is the key [3]. Because of the large amounts of data from the measured graphics or waveform, they are too difficult to recognize directly. So they need to be transformed from original data to signal features. So far, researchers from home and abroad use statistical characteristic parameters, pulse characteristic parameters, moment features, or fractal characteristics to recognize discharge types. Because of their accuracy and less characteristic parameters, fractal characteristics are becoming more and more popular [4].
Back propagation (BP) network is a kind of widely used pattern recognizer [5]. It has some disadvantages such as network structure, local minimum, and over- or underlearning. Support Vector Machine (SVM) is a new kind of pattern recognition method which is proposed and developed in the recent decades. It can solve the small sample learning problems and solve the local minimum and over-studylearning or under-study-learning problems. Now the SVM has been widely used in the power system [6].

\section{Support Vector Machine}

SVM is a kind of data mining method based on statistical learning theory. It can handle the regression problems (time series analysis) and pattern recognition (classification problem and discriminant analysis) successfully [7].

The principle of SVM is to find an optimized classified hyperplane based on classification requirements. The hyperplane can maximize blank area on both its sides and, at the same time, guarantee the classification accuracy [8]. In theory, SVM can achieve optimal classification. 
Assuming that the given samples are $\left\{\left(x_{1}, y_{1}\right),\left(x_{2}\right.\right.$, $\left.\left.y_{2}\right), \ldots,\left(x_{l}, y_{l}\right)\right\}$ and $x_{i} \in X \subseteq R^{n}, y_{i} \in X \subseteq R^{n}$. The number of samples is $l$. SVM defines a nonlinear feature space mapping function by inner product function $\varphi(\cdot)$.

The samples $x$ are mapped into a high-dimensional space $H$. In the high-dimensional space, the linear regression function is constructed based on the principle of structural risk minimization [9]:

$$
f(x)=\omega \cdot \phi(x)+b
$$

where $\omega$ and $b$ are the weight coefficient and the deviation, which can be obtained by minimizing the objective function as follows:

$$
\min R(\omega)=\frac{1}{l} \sum_{i=1}^{l}\left|y_{i}-f\left(x_{i}\right)\right|_{\varepsilon}+C\|\omega\|^{2},
$$

where $C$ is the generalization constant.

When using SVM, solving regression problem, introduce linear insensitive loss function $\varepsilon$. Cost function $|\cdot|_{\varepsilon}$ is Vapnik insensitive loss function [10]:

$$
\left|y_{i}-f\left(x_{i}\right)\right|_{\varepsilon}= \begin{cases}\left|y_{i}-f\left(x_{i}\right)\right|-\varepsilon & \left|y_{i}-f\left(x_{i}\right)\right|<\varepsilon \\ 0 & \text { others. }\end{cases}
$$

Considering the tolerated fitting deviation, the original problem can be transformed into structural risk minimization objective function problem by introducing two groups of nonnegative slack variables and using the principle of structural risk minimization. The optimization problem as (1) is transformed into a constrained minimization problem as follows:

$$
\min \frac{1}{2}\|\omega\|^{2}+C \frac{1}{l} \sum_{i=1}^{l}\left(\xi+\xi^{*}\right) .
$$

Constraints are

$$
\begin{aligned}
& y_{i}-\omega \cdot \phi\left(x_{i}\right)-b \leq \varepsilon+\xi \\
& \begin{aligned}
\omega \cdot \phi\left(x_{i}\right)+b-y_{i} \leq & \varepsilon+\xi^{*} \\
& \xi \geq 0, \xi^{*} \geq 0, i=1,2, \ldots, l-1, l .
\end{aligned}
\end{aligned}
$$

Then, build Lagrange function and transform inequality constraints into equality constraints as follows:

$$
\begin{aligned}
L= & \frac{1}{2}\|\omega\|^{2}+C \sum_{i=1}^{l}\left(\xi_{i}+\xi_{i}^{*}\right)-\sum_{i=1}^{l}\left(\eta_{i} \xi_{i}+\eta_{i}^{*} \xi_{i}^{*}\right) \\
& -\sum_{i=1}^{l} \alpha_{i}\left(\varepsilon+\xi_{i}-y_{i}+\omega \cdot \phi\left(x_{i}\right)+b\right) \\
& -\sum_{i=1}^{l} \alpha_{i}^{*}\left(\varepsilon+\xi_{i}^{*}-y_{i}+\omega \cdot \phi\left(x_{i}\right)+b\right),
\end{aligned}
$$

where $\eta_{i}, \eta_{i}^{*}, \alpha_{i}, \alpha_{i}^{*}$ is Lagrange multipliers and it satisfies the nonnegative constraints as well:

$$
\eta_{i}, \eta_{i}^{*}, \alpha_{i}, \alpha_{i}^{*} \geq 0
$$

The optimization problem as (6) can be solved in its dual form. According to the Karush-Kuhn-Tucker (KKT) conditions, the original problem can be transformed into the optimization objective function as follows [11]:

$$
\begin{aligned}
& W\left(\alpha_{i}, \alpha_{i}^{*}\right) \\
& =\sum_{i=1}^{l} y_{i}\left(\alpha_{i}-\alpha_{i}^{*}\right)-\varepsilon \sum_{i=1}^{l}\left(\alpha_{i}+\alpha_{i}^{*}\right) \\
& \quad-\frac{1}{2} \sum_{i=1}^{l} \sum_{i=1}^{l}\left(\alpha_{i}-\alpha_{i}^{*}\right)\left(\alpha_{j}-\alpha_{j}^{*}\right)\left(\phi\left(x_{i}\right) \cdot \phi\left(x_{j}\right)\right) .
\end{aligned}
$$

Constraints are

$$
\sum_{i=1}^{l}\left(\alpha_{i}-\alpha_{i}^{*}\right)=0, \quad \alpha_{i}, \alpha_{i}^{*} \in[0, C]
$$

Maximize (8) as

$$
f(x)=\sum_{i=1}^{l}\left(\alpha_{i}-\alpha_{i}^{*}\right)\left(\phi\left(x_{i}\right) \cdot \phi(x)\right)+b,
$$

where $\alpha_{i}, \alpha_{i}^{*}$ is the introduced nonnegative Lagrange multiplier, $K\left(x_{i}, x_{j}\right)$ is the kernel function based on Mercer condition, and the inner product kernel function is as follows [12]:

$$
K\left(x_{i}, x\right)=\phi\left(x_{i}\right) \cdot \phi(x) .
$$

The introduction of kernel function takes the place of dot product in the high-dimensional space, avoiding the problem of nonlinear mapping function which reduces the computation and complexity significantly. Then the result is as follows:

$$
f(x)=\sum_{i=1}^{l}\left(\alpha_{i}-\alpha_{i}^{*}\right) \cdot K\left(x_{i}, x\right)+b .
$$

\section{Fractal Method}

Most objects in nature are very complex and irregular. When the object has some similarities between the local and global, it can be viewed as fractal. The fractal dimension, as quantitative characterization and basic parameter of the fractal, is an important principle of the fractal theory. According to different definitions and calculation methods, box dimension and information dimension are often used in the fractal calculation [13].

To the Point Set $\Omega \subset R_{n}$, if it can be covered by $N(r) n$ dimensional hypercube whose side length is $r$, then the box dimension of the Point Set is

$$
D_{B}=\lim _{r \rightarrow 0} \frac{\ln N(r)}{\ln (1 / r)} .
$$

Because the box dimension is not able to reflect the unevenness of geometric objects, a box with one or several points may have the same weights. The information 


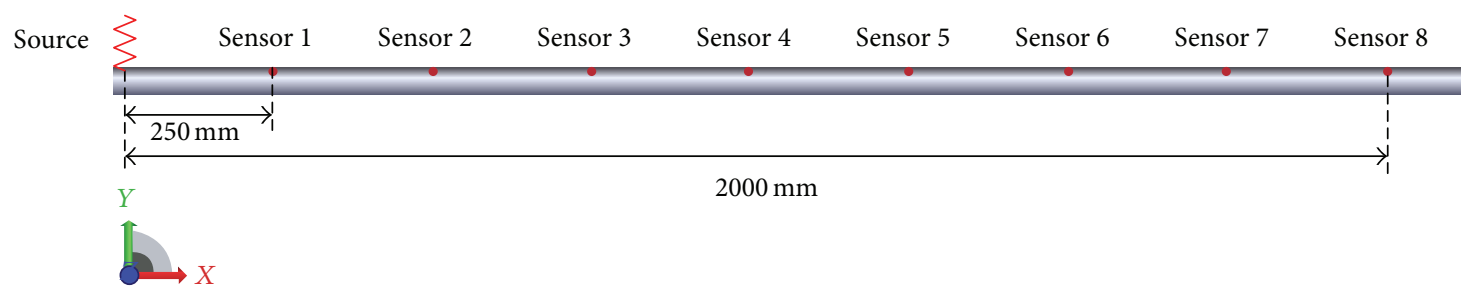

FIGURE 1: XLPE cable simulation model and the distribution of the sensors.

dimension has some advantages in this situation [14]. If the possibility of the Set Point falling into the $k$ th hypercubes is $P_{k}(r)$, define the number of information as entropy, which can accurate the system status to level $r$, then

$$
I(r)=-\sum_{k=1}^{N(r)} P_{k}(r) \ln P_{k}(r) .
$$

So, the information dimension of Point Set $\Omega$ is

$$
D_{I}=\lim _{r \rightarrow 0} \frac{I(r)}{\ln (1 / r)} .
$$

\section{Simulations}

4.1. Finite Difference Time Domain (FDTD). Finite Difference Time Domain (FDTD) is a numerical calculation method in solving the time domain electromagnetic problem. It finishes finite difference discretization in time and space domain based on Maxwell equations and then builds the central finite difference equations whose accuracy is second-order. FDTD can simulate any kind of electromagnetic structure according to the electromagnetic parameters and medium parameters of the model [15]. In isotropic media, two curl equations of Maxwell equations are

$$
\begin{aligned}
& \nabla \times \vec{E}=-\mu \frac{\partial \vec{H}}{\partial t}-\sigma^{*} \vec{H}, \\
& \nabla \times \vec{H}=\varepsilon \frac{\partial \vec{E}}{\partial t}+\sigma \vec{E},
\end{aligned}
$$

where $\varepsilon$ is the permittivity, $\mathrm{F} \cdot \mathrm{m}^{-1} ; \mu$ is the magnetic permeability, $\mathrm{H} \cdot \mathrm{m}^{-1} ; \sigma$ is the conductivity, $\mathrm{S} \cdot \mathrm{m}^{-1}$; and $\sigma^{*}$ is the magnetoconductivity, $\Omega \cdot \mathrm{m}^{-1}$.

In suitable boundary conditions and initial conditions, FDTD can give the time domain characteristic of electromagnetic wave by solving the differential Maxwell equations, which makes it easier for us to analyze the discharge problems in the XLPE power cables [16].

4.2. Model of XLPE Cables. XLPE cables have the typical coaxial structure. To find out the characteristics of this structure, FDTD simulation program is applied. Figure 2 shows the model of the real $30 \mathrm{kV}$ XLPE cable. The partial discharges source located in the cavity $1 \mathrm{~mm}$ from the left side of the port is a discharge voltage pulse along the radial distribution of the cavity. In the coaxial cavity, we placed sensors every $250 \mathrm{~mm}$ (red points in Figure 1).

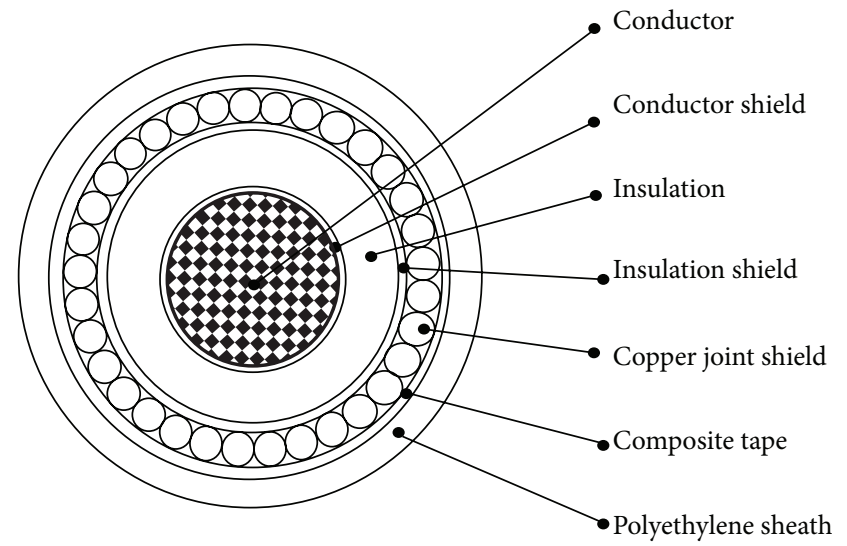

Figure 2: Structure of the model.

The structure and the materials electrical characteristics of the model are shown as in Figure 2 and Table 1.

4.3. Partial Discharge Source. Gaussian pulse is used to simulate the partial discharge voltage pulse:

$$
u(t)=U_{0} e^{-\left(t-t_{0}\right)^{2} / 2 \sigma^{2}},
$$

where $U_{0}$ is the amplitude of the pulse voltage; $t_{0}$ represents the time-delay constant; and $\sigma$ is the decay time constant. Assuming that the amplitude of the voltage pulse is $1000 \mathrm{~V}$, $\sigma=0.17 \mathrm{~ns}$, and $t_{0}=5 \mathrm{~ns}$, Figure 3 shows the pulse voltage waveform, where the rise time $t_{r}$ of pulse is $2 \mathrm{~ns}$, the fall time $t_{f}$ is $2 \mathrm{~ns}$, and the pulse width $t_{w}$ is $5 \mathrm{~ns}$.

4.4. Simulation Results. In order to study propagation characteristics of the electromagnetic signal in the coaxial waveguide, signals are taken from sensor 1 to sensor 8 . Field signal which only contains TEM wave measured by sensor 1 is shown in Figure 4.

Results show that the propagation of the signal from sensor 1 to sensor 8 has caused its amplitude to weaken from $9799.4 \mathrm{mV} / \mathrm{m}$ to $7485.6 \mathrm{mV} / \mathrm{m}$, reduced by $2.34 \mathrm{~dB}$, and the signal waveform is changed significantly. However, the amplitude and waveform of the signal components below $400 \mathrm{MHz}$ which only contain TEM wave are not changed. It indicates that when propagating in the coaxial waveguide, the attenuation of TEM wave is small and its waveform will remain basically unchanged, while the amplitude of electromagnetic wave decays a lot and the waveform changes because of the dispersion effect of higher-order mode waves. 
TABLE 1: Materials electrical characteristics of the model.

\begin{tabular}{lcccc}
\hline Structure parts & Outer diameter $/ \mathrm{mm}$ & Material & Relative permittivity & Conductivity \\
\hline Conductor & 20.4 & Copper & - & 30.0 \\
Conductor shield & 21.8 & Semiconductor & 3.2 & 2 \\
Insulation & 30.1 & Silicone rubber & 90.0 & 0 \\
Insulation shield & 31.5 & Conductive silicon rubber & - & 0.5 \\
Copper joint shield & 34.9 & Copper & 3.0 & $5.8 \times 10^{7}$ \\
Composite tape & 35.5 & PVC & 2.3 & $1 \times 10^{-13}$ \\
Polyethylene sheath & 40.0 & XLPE & 0 \\
\hline
\end{tabular}

TABLE 2: Real data of discharge signals recorded.

\begin{tabular}{|c|c|c|c|}
\hline & Information dimension & Box dimension & Discharge type \\
\hline Discharge 1 & $(0.51,1.52)$ & $(0.35,1.86)$ & Creeping discharge \\
\hline Discharge 2 & $(1.42,1.15)$ & $(1.67,0.85)$ & External corona discharge \\
\hline Discharge 3 & $(1.63,1.63)$ & $(1.44,1.35)$ & Floating electrode discharge \\
\hline Discharge 4 & $(1.69,1.06)$ & $(1.81,0.77)$ & External corona discharge \\
\hline Discharge 5 & $(0.46,1.78)$ & $(0.45,1.55)$ & Creeping discharge \\
\hline Discharge 6 & $(0.55,1.65)$ & $(0.37,1.61)$ & Creeping discharge \\
\hline Discharge 7 & $(0.61,1.14)$ & $(0.77,1.27)$ & Internal air discharge \\
\hline Discharge 8 & $(0.75,1.02)$ & $(0.65,1.18)$ & Internal air discharge \\
\hline Discharge 9 & $(1.77,1.67)$ & $(1.27,1.54)$ & Floating electrode discharge \\
\hline Discharge 10 & $(0.47,1.68)$ & $(0.28,1.61)$ & Creeping discharge \\
\hline
\end{tabular}

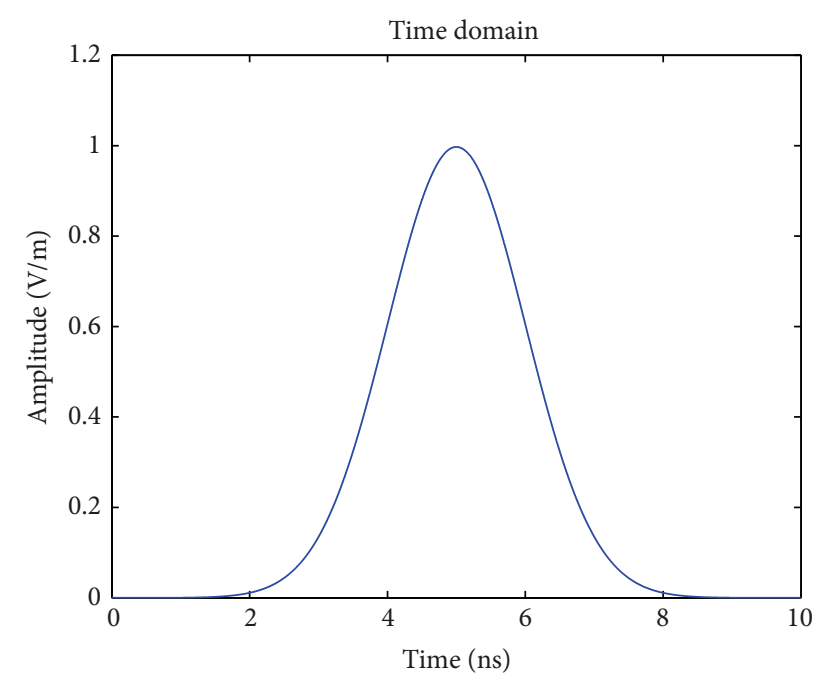

FiguRE 3: Waveform of partial discharge voltage pulse.

4.5. Real Data of Discharge Signals Recorded in $30 \mathrm{kV}$ Power Cable. The simulation data may have some deviation. In order to make the result more accurate, a list of discharge signals from a real power cable line are monitored and recorded. The data recording system is shown as Figure 5.

The band of the measuring impedance is $45 \sim 250 \mathrm{kHz}$, and the setting of the monitor is $500 \mathrm{kS} / \mathrm{s}$ with $1 \mathrm{MB}$ memory capacity and threshold trigger. So the monitor can store up to 100 cycles of discharge waveform. Their information dimension and box dimension are calculated and recorded, as shown in Table 2.

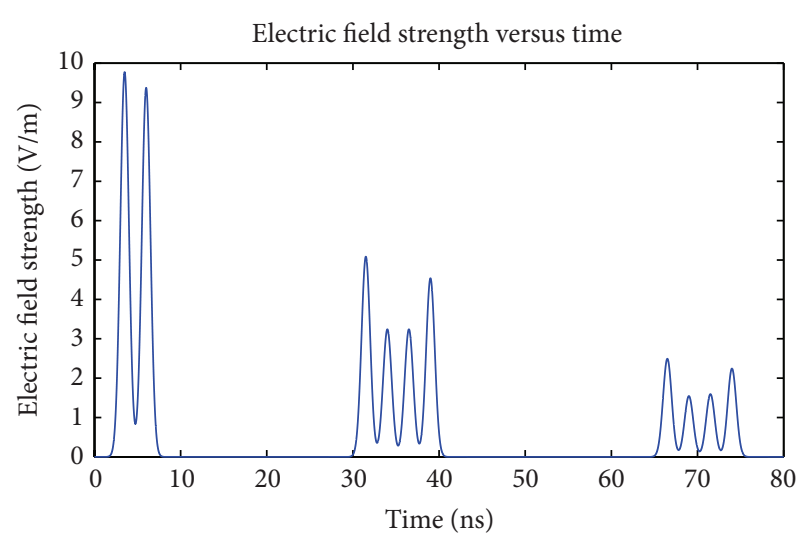

FIgURE 4: Waveform of sensor 1.

\section{Training and Recognition Based on Multi-SVM}

In order to make the multi-SVM recognizer more accurate, the simulation data are used as training samples to train the SVM and the measured data are used as testing samples to verify its accuracy.

When training SVM recognizer, SVM model and its Lagrange multipliers can be determined by training the samples and solving the quadratic programming equations. The flowchart of multi-SVM recognizer is shown as Figure 6.

5.1. Set Typical Discharge in Simulation to Get Training Samples for Multi-SVM. Studies show that there are approximately four types of PD in XLPE cables: creeping 


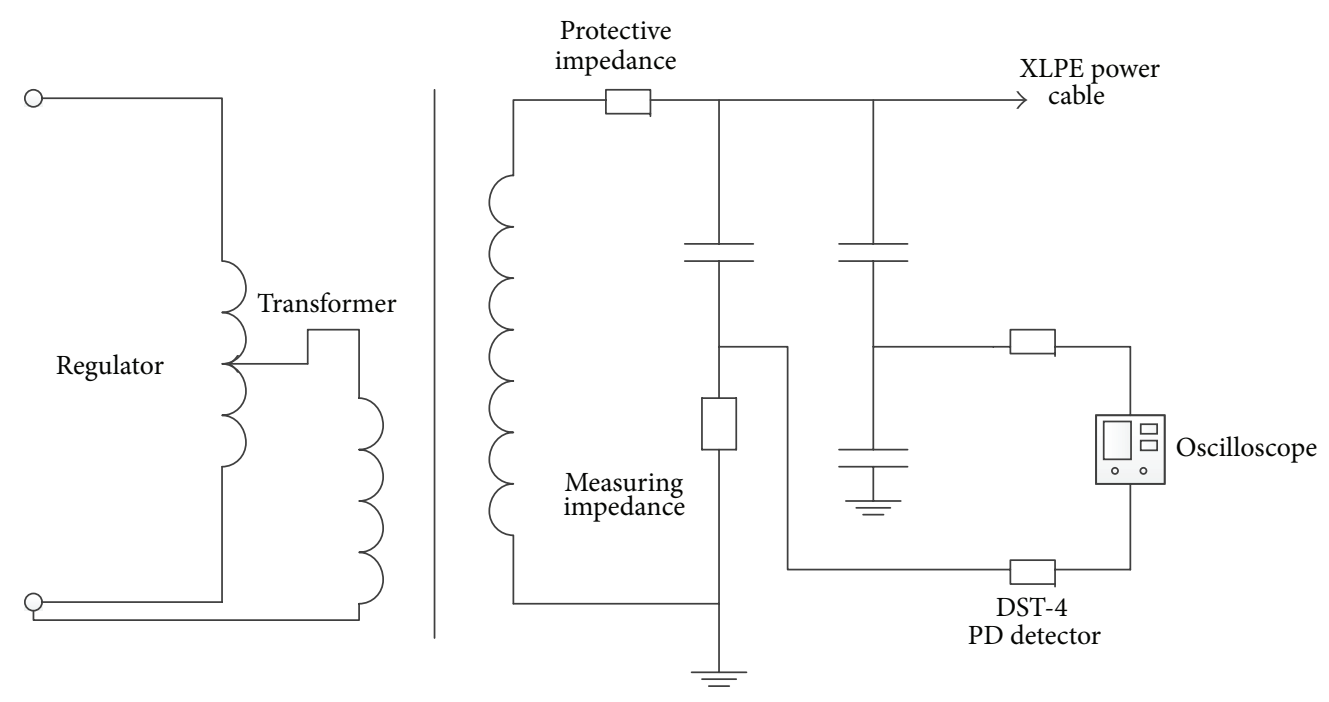

FIGURE 5: Real data of discharge signal recording system.

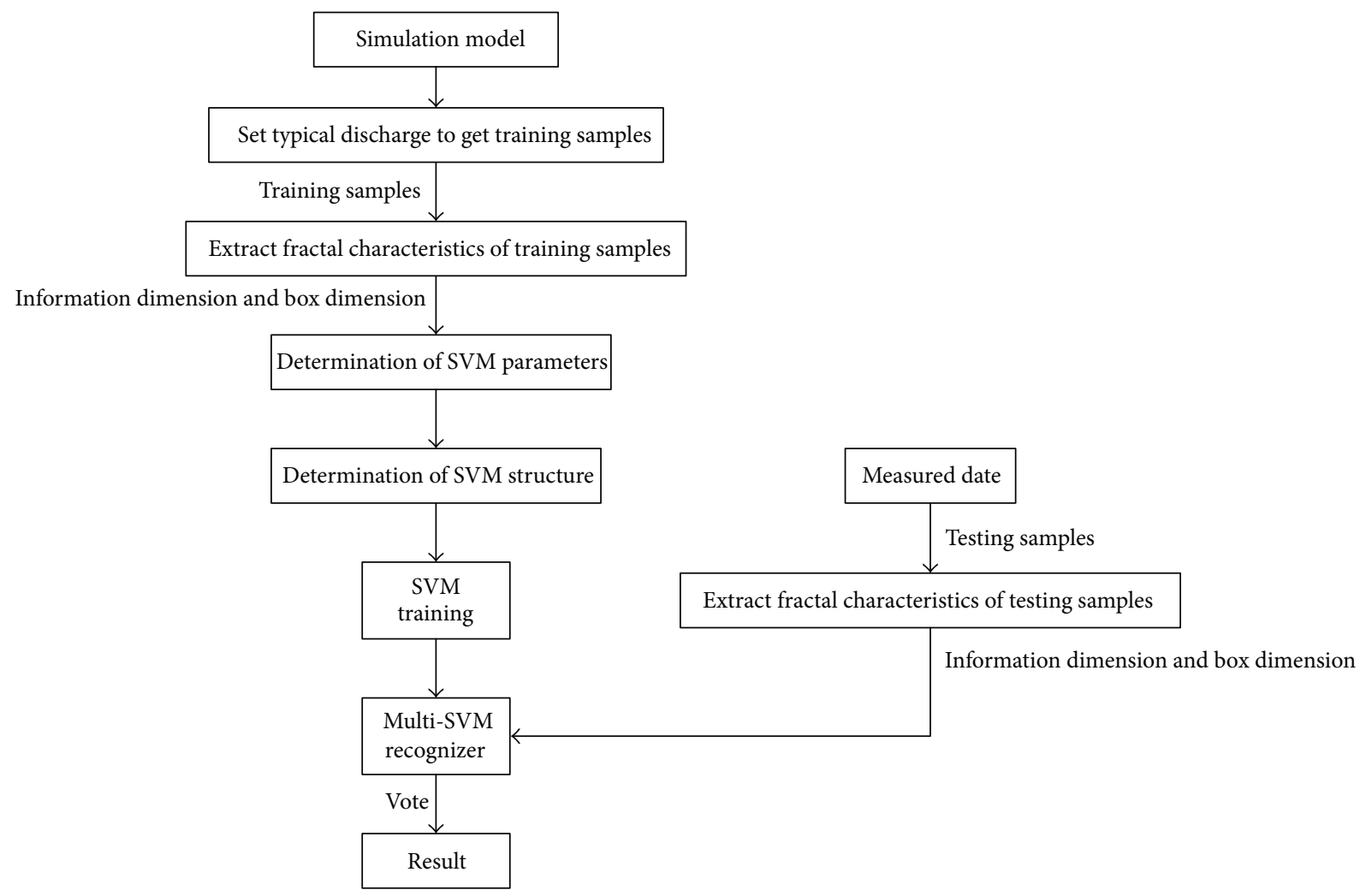

FIGURE 6: Flowchart of SVM recognizer.

discharge, floating electrode discharge, internal air discharge, and external corona discharge. According to their different characteristics, four PD faults are set in the simulation model.

(1) Creeping Discharge Model. To simulate the creeping discharge, a long thin wire is fixed on the joint surface of the main insulation and silicone rubber. Its length is $30 \mathrm{~mm}$ and its diameter is $0.5 \mathrm{~mm}$.
(2) Floating Electrode Discharge Model. To simulate the floating electrode discharge model, a small round copper is fixed between the XLPE insulation of cable accessories and the main insulation. It has a diameter of $12 \mathrm{~mm}$ and a thickness of $0.5 \mathrm{~mm}$.

(3) Internal Air Discharge Model. To simulate the internal air discharge model, a needle is fixed between the metal 


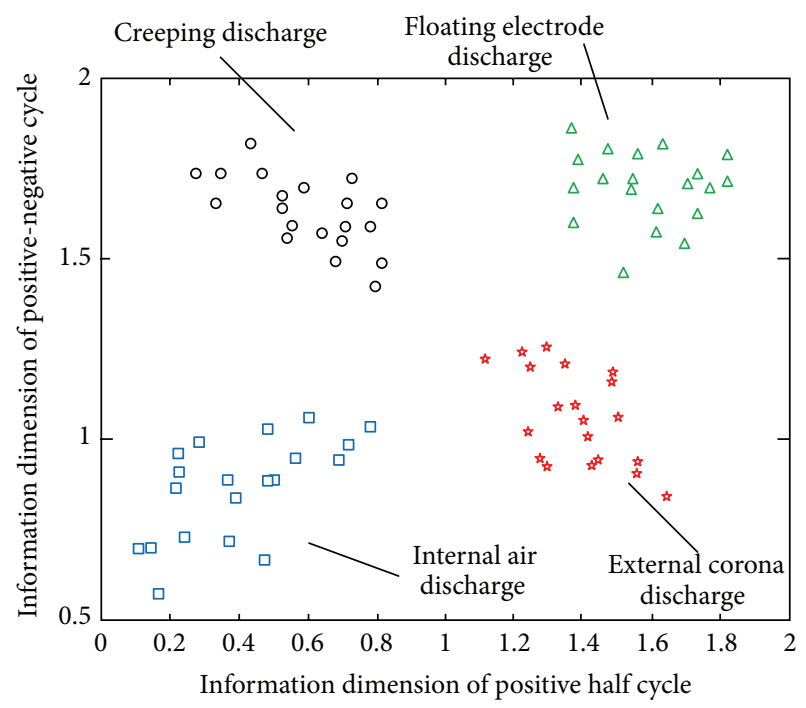

(a) Information dimension

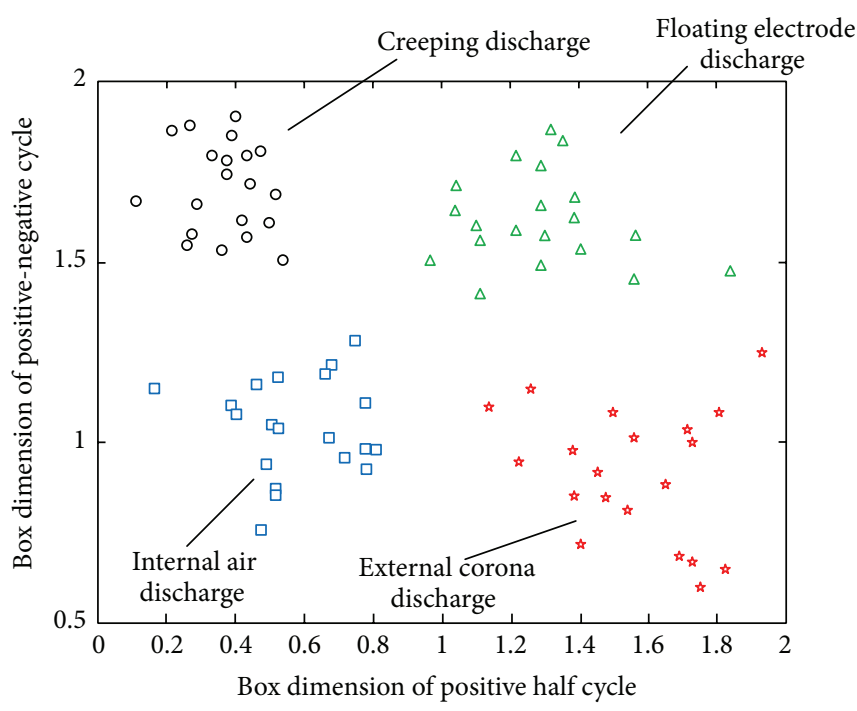

(b) Box dimension

FIGURE 7: Fractal characteristics of partial discharge.

shield and the outer semiconductor layer. It has a diameter of $0.2 \mathrm{~mm}$ and a length of $2 \mathrm{~mm}$.

(4) External Corona Discharge Model. To simulate the external corona discharge model, a needle is fixed on the high voltage conductor. It has a diameter of $0.1 \mathrm{~mm}$ and a length of $10 \mathrm{~mm}$.

5.2. Extract Fractal Characteristics of Training Samples. Gather 80 groups of data (20 groups of each discharge type) from cable partial discharge simulations and process them according to the minimum box counting method. Four fractal characteristics are found from grayscale images: box dimension of the positive half cycle $D_{B}^{+}$, box dimension of the negative half cycle $D_{B}^{-}$, information dimension of the positive half cycle $D_{I}^{+}$, and information dimension of the negative half cycle $D_{I}^{-}$. The results are shown as in Figure 7.

Figure 7 shows box dimension and information dimension of different types of discharge gathered in different areas. So fractal characteristics such as box dimension and information dimension have a strong ability to distinguish between different types of discharges [17, 18].

5.3. Determination of Multi-SVM Parameters. As we know, it is difficult for SVM to solve partial discharge problems. So in this paper the algorithm proposed is multi-SVM which is made up of six SVM. The principle to build multi-SVM recognizer proposed in this paper is to combine six one-toone SVM together.

Use RBF as multi-SVM method's kernel function:

$$
K(x, y)=\exp \left(-\frac{\|x-y\|_{2}^{2}}{\sigma^{2}}\right) .
$$

5.4. Determination of Multi-SVM Structure. Comparatively speaking, there are two different kinds of branching algorithms: one-to-one algorithms and one-to-many algorithms [19]. For one-to-one algorithm, each SVM recognizer only has two kinds of status [20]. So, one-to-one algorithm is easy to be trained and its decision boundary is relatively simple. Its classification accuracy is much better than the one-to-many algorithm as well. Therefore, one-to-one algorithm is used in this paper.

Six one-to-one SVM are proposed in this paper to recognize four types of discharges. They are defined as SVM1, SVM2, SVM3, SVM4, SVM5, and SVM6. The multi-SVM recognizer proposed in this paper is made up of these six SVM. The discharge type is determined by the vote shown in Table 3. The vote results of four discharge types are shown as in Table 4.

5.5. SVM Training and Recognizer Structure. Use the 80 groups of data (20 groups of each discharge type) from cable partial discharge simulations to train the SVM recognizer. After training, the number of support vectors of each SVM is shown as in Table 5.

Take SVM2 as an example. The structure of SVM2 is shown as in Figure 8. $x_{1}, x_{2}, \ldots, x_{18}$ is the support vectors of SVM 2 and $\alpha_{1}-\alpha_{1}^{*}, \alpha_{2}-\alpha_{2}^{*}, \ldots, \alpha_{18}-\alpha_{18}^{*}$ is the weights shown as Table 6 .

5.6. Extract Fractal Characteristics of Testing Samples. Use the 10 groups of real data recorded in $30 \mathrm{kV}$ power cable as testing samples to verify the effectiveness of the multi-SVM recognizer proposed in this paper. After normalizing the data, the fractal characteristics of testing samples are shown as in Figure 9.

5.7. Vote Results. After inputting the fractal characteristics of the testing samples into the multi-SVM, the vote results are shown as in Table 7. 
TABle 3: Determine the discharge type by vote.

\begin{tabular}{lcccc}
\hline Recognizer & Creeping discharge & Floating electrode discharge & Internal air discharge & External corona discharge \\
\hline SVM1 & 0 & 0 & 1 & -1 \\
SVM2 & 1 & 0 & -1 & 0 \\
SVM3 & 1 & 0 & 0 & -1 \\
SVM4 & 0 & 1 & -1 & 0 \\
SVM5 & 0 & 1 & 0 & -1 \\
SVM6 & 1 & -1 & 0 & 0 \\
\hline
\end{tabular}

TABLE 4: Vote results of four discharge types.

\begin{tabular}{lc}
\hline Discharge type & Vote results \\
\hline Creeping discharge & SVM1 + SVM2 + SVM3 + SVM4 + SVM5 + SVM6 \\
Floating electrode discharge & SVM1 + SVM2 + SVM3 + SVM4 + SVM5 - SVM6 \\
Internal air discharge & SVM1 - SVM2 + SVM3 - SVM4 + SVM5 + SVM6 \\
External corona discharge & $-S V M 1+$ SVM2 - SVM3 + SVM4 - SVM5 + SVM6 \\
\hline
\end{tabular}

TABLE 5: The number of support vectors.

\begin{tabular}{lccccc}
\hline SVM1 & SVM2 & SVM3 & SVM4 & SVM5 & SVM6 \\
\hline 40 & 18 & 23 & 19 & 35 & 24 \\
\hline
\end{tabular}

Compared with Table 2, all the recognition results from multi-SVM are correct. So the multi-SVM recognizer proposed in this paper is effective in recognizing the discharge type of $30 \mathrm{kV}$ XLPE power cables.

5.8. Accuracy Comparison. There are many kinds of intelligent algorithm such as BP neural networks and ELM. In order to prove the accuracy of multi-SVM, systematical and comprehensive comparisons are made in this paper. BP neural networks and ELM are applied into the recognizer instead of multi-SVM to recognize the discharge type.

5.8.1. BP Neural Networks Recognizer. BP neural networks are widely used in many aspects. In this paper, calculate the fractal calculation parameters $\left(D_{B}^{+}, D_{B}^{-}, D_{I}^{+}, D_{I}^{-}\right)$of discharge waveform from simulation as the BP neural network input vector $X$; the discharge type recognized is the output $Y$ of the BP neural network. The hidden layer is a single neuron structure, which uses supervised learning method. The number of hidden layer neurons adjusts in the learning process and finally get the best BP neural network recognizer model.

The number of input layer neurons is $k$, the number of hidden layer neurons is $g$, and the number of output layer neurons is $m$. Use standard hyperbolic tangent function as the transfer function of the hidden layer. The details of BP neural network are shown in Table 8. The structure of BP neural network is shown in Figure 10.

In the setting, the target error is $10^{-4}$; after 400 times of training, the BP neural network finishes. The dropping of deviation by training is shown as in Figure 11. Finally, the number of input layer neurons is $k=6$, the number of

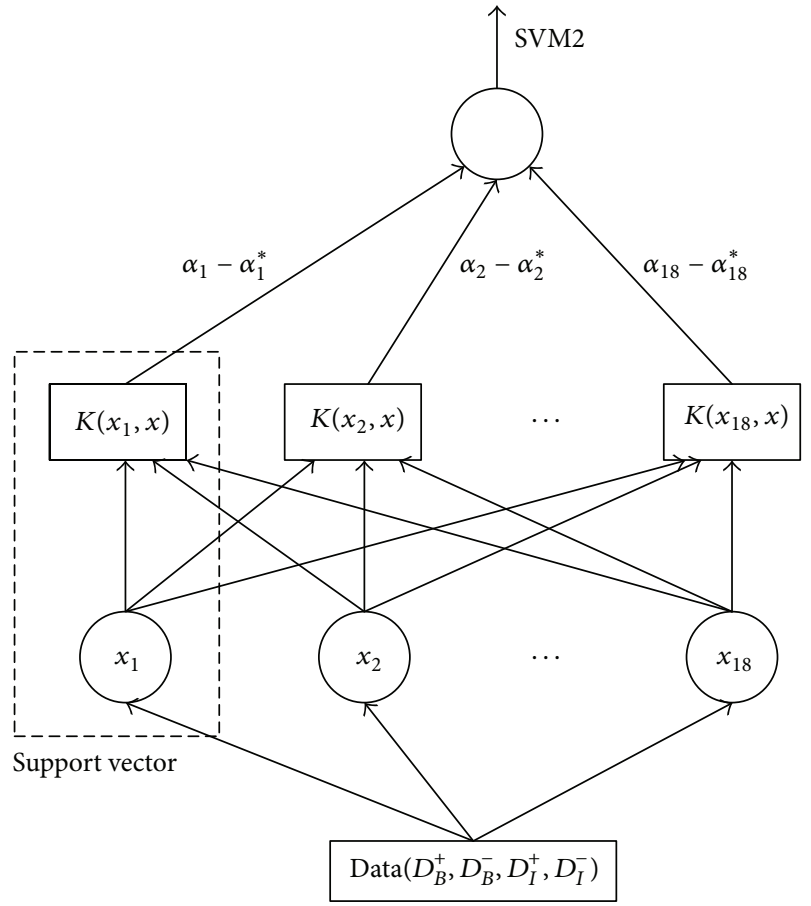

FIGURE 8: The structure of SVM2.

hidden layer neurons is $g=9$, and the number of output layer neurons is $m=1$.

Use 100 groups of simulation data to test the accuracy of BP neural network; the results are shown as in Table 9.

5.8.2. ELM Recognizer. In the BP neural networks, the parameters of hidden layer are determined by large numbers of iterations which will take a lot of time and the results may be unsatisfying as well. In order to improve the performance of the network, ELM is proposed by Huang G.B. Etc [21-23]. Extreme Learning Machine (ELM) is a fast training algorithm for networks with single hidden layer. Its parameters of 
TABLE 6: Weights of support vectors of SVM2.

\begin{tabular}{lcccccccc}
\hline$\alpha_{1}-\alpha_{1}^{*}$ & $\alpha_{2}-\alpha_{2}^{*}$ & $\alpha_{3}-\alpha_{3}^{*}$ & $\alpha_{4}-\alpha_{4}^{*}$ & $\alpha_{5}-\alpha_{5}^{*}$ & $\alpha_{6}-\alpha_{6}^{*}$ & $\alpha_{7}-\alpha_{7}^{*}$ & $\alpha_{8}-\alpha_{8}^{*}$ & $\alpha_{9}-\alpha_{9}^{*}$ \\
\hline 1.32 & 0.52 & 0.48 & 0.74 & 0.14 & 0.96 & 1.25 & 0.36 \\
\hline$\alpha_{10}-\alpha_{10}^{*}$ & $\alpha_{11}-\alpha_{11}^{*}$ & $\alpha_{12}-\alpha_{12}^{*}$ & $\alpha_{13}-\alpha_{13}^{*}$ & $\alpha_{14}-\alpha_{14}^{*}$ & $\alpha_{15}-\alpha_{15}^{*}$ & $\alpha_{16}-\alpha_{16}^{*}$ & $\alpha_{17}-\alpha_{17}^{*}$ & $\alpha_{18}-\alpha_{18}^{*}$ \\
\hline 0.26 & 0.44 & 0.61 & 1.73 & 0.81 & 0.41 & 0.52 & 1.16 & 0.85 \\
\hline
\end{tabular}

TABLE 7: Vote results of testing samples.

\begin{tabular}{lccccc}
\hline & $\begin{array}{c}\text { Creeping } \\
\text { discharge }\end{array}$ & Floating electrode discharge & Internal air discharge External corona discharge & Discharge type result \\
\hline Discharge 1 & $\mathbf{3}$ & 0 & 0 & 0 & Creeping discharge \\
Discharge 2 & 0 & 0 & 0 & $\mathbf{3}$ & External corona discharge \\
Discharge 3 & 0 & $\mathbf{3}$ & 0 & 0 & Floating electrode discharge \\
Discharge 4 & 0 & 0 & 0 & 0 & External corona discharge \\
Discharge 5 & $\mathbf{3}$ & 0 & 0 & 0 & Creeping discharge \\
Discharge 6 & $\mathbf{3}$ & 0 & 0 & 0 & Creeping discharge \\
Discharge 7 & 0 & 0 & 3 & 0 & Internal air discharge \\
Discharge 8 & 0 & 0 & 3 & 0 & Internal air discharge \\
Discharge 9 & 0 & $\mathbf{3}$ & 0 & 0 & Floating electrode discharge \\
Discharge 10 & $\mathbf{3}$ & 0 & 0 & Creeping discharge \\
\hline
\end{tabular}

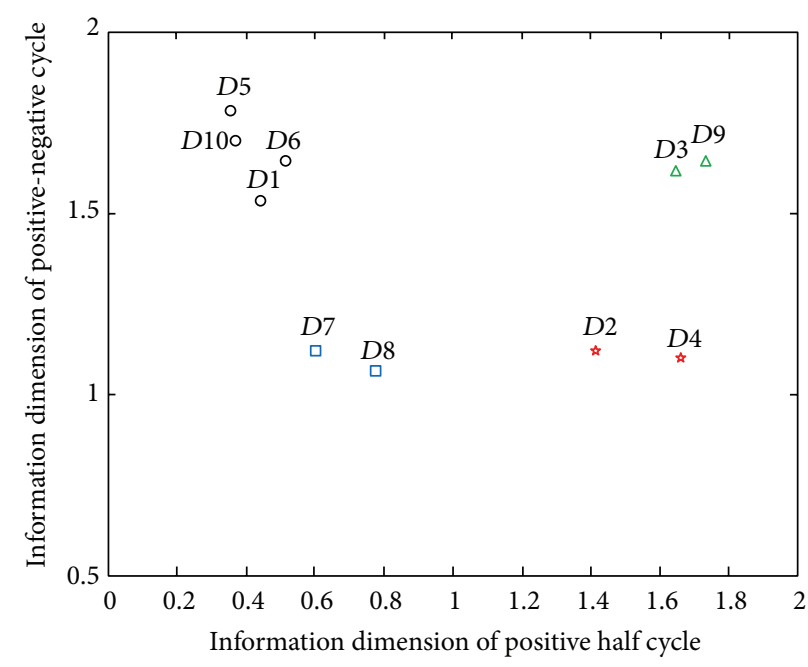

(a) Information dimension

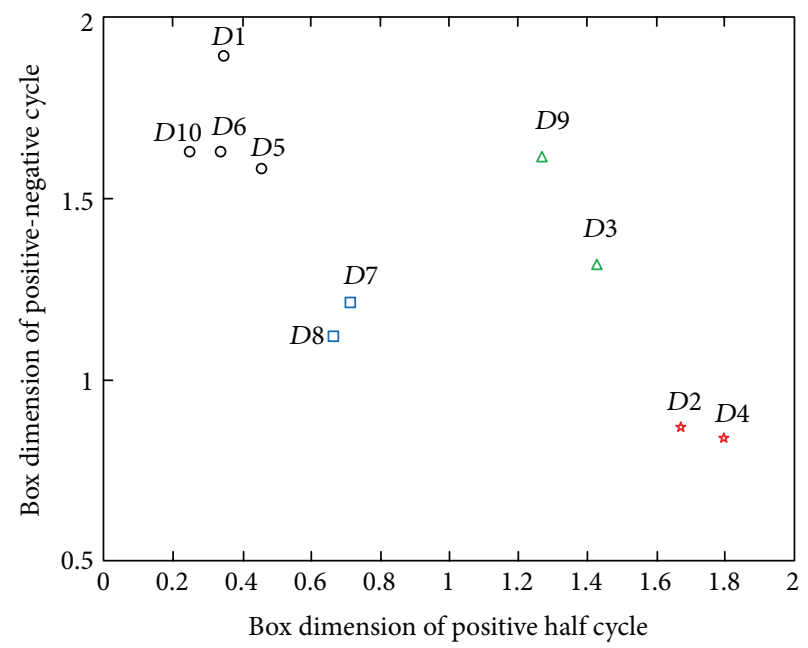

(b) Box dimension

FIGURE 9: Fractal characteristics of testing samples.

hidden layer are determined randomly without any iteration, which significantly reduce the adjusting time for network parameters. The speed of training increases greatly and the results are better.

\section{ELM Algorithm}

(a) Input: training samples made up of fractal calculation parameters $\left(D_{B}^{+}, D_{B}^{-}, D_{I}^{+}, D_{I}^{-}\right)$of discharge signals; transfer function of the hidden layer; number of input layer neurons $L$.

(b) Parameters of hidden layer generated randomly $\left(a_{i}, b_{i}\right) i=1, \ldots, L$. (c) Calculating hidden layer output matrix $H$ ( $H$ must be full rank).

(d) Output: optimized weight of network $\beta$ : $\beta=H * T$.

Use 100 groups of simulation data to test the accuracy of ELM; the results are shown as in Table 10.

5.8.3. Multi-SVM Recognizer. In order to test the accuracy of the multi-SVM recognizer proposed in this paper, use 100 groups of simulation data to test the accuracy of multi-SVM; the results are shown as in Table 11. 
TABLE 8: Detailed expression of BP neural networks.

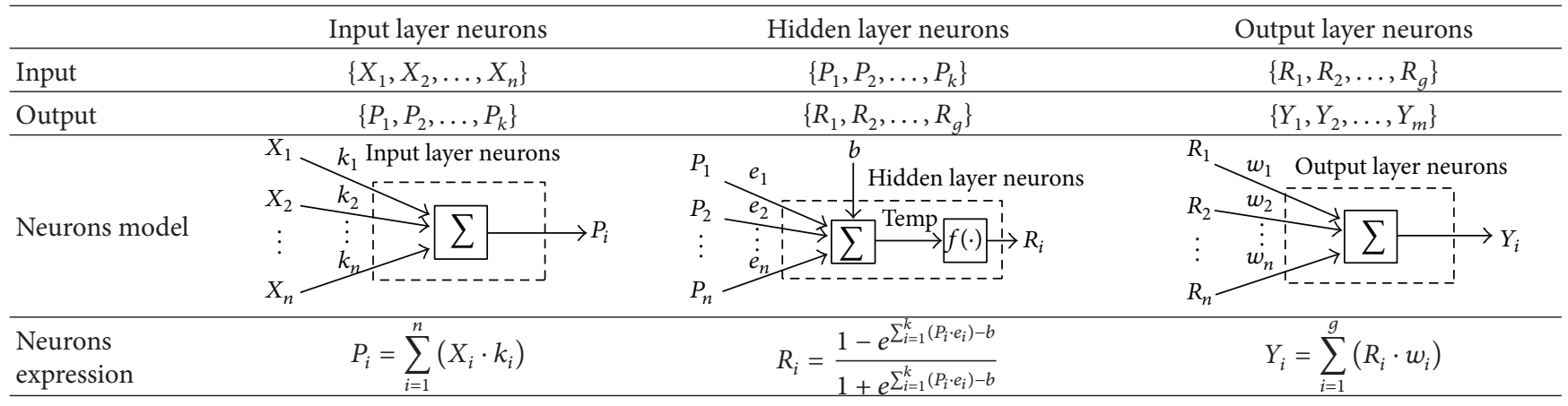

TABLE 9: Results of BP neural networks.

\begin{tabular}{lcccc}
\hline & & \multicolumn{2}{c}{ Discharge type } \\
& Creeping discharge & Floating electrode discharge & Internal air discharge & External corona discharge \\
\hline Test sample number & 100 & 100 & 100 & 100 \\
Correct results number & 78 & 76 & 80 & 75 \\
Accuracy & $78 \%$ & $76 \%$ & $80 \%$ & $75 \%$ \\
\hline
\end{tabular}

TABLE 10: Results of ELM.

\begin{tabular}{lcccc}
\hline & & \multicolumn{2}{c}{ Discharge type } \\
& Creeping discharge & Floating electrode discharge & Internal air discharge & External corona discharge \\
\hline Test sample number & 100 & 100 & 100 & 100 \\
Correct results number & 90 & 88 & 91 & 86 \\
Accuracy & $90 \%$ & $88 \%$ & $91 \%$ & $86 \%$ \\
\hline
\end{tabular}

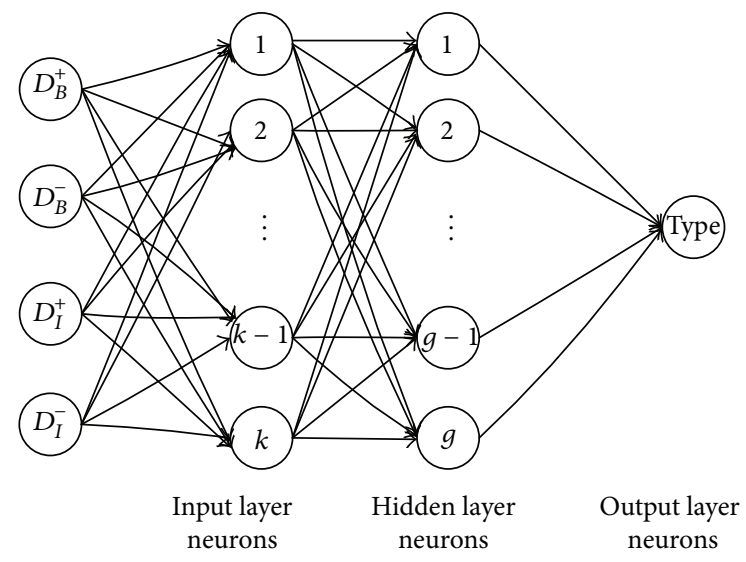

FIGURE 10: Structure of BP neural networks.

5.8.4. Comparison. The accuracy comparison between BP neural networks, SLM, and SVM is shown as in Table 12. Compared with BP neural networks, ELM and multi-SVM both perform excellently (accuracy of each type over $85 \%$ ). As a matter of fact, from the view of accuracy, multi-SVM recognizer is even better (accuracy of each type over 90\%).

The effectiveness of multi-SVM recognizer is satisfied. The accuracy of multi-SVM recognizer of all types is over $90 \%$, especially the recognition of internal air discharge

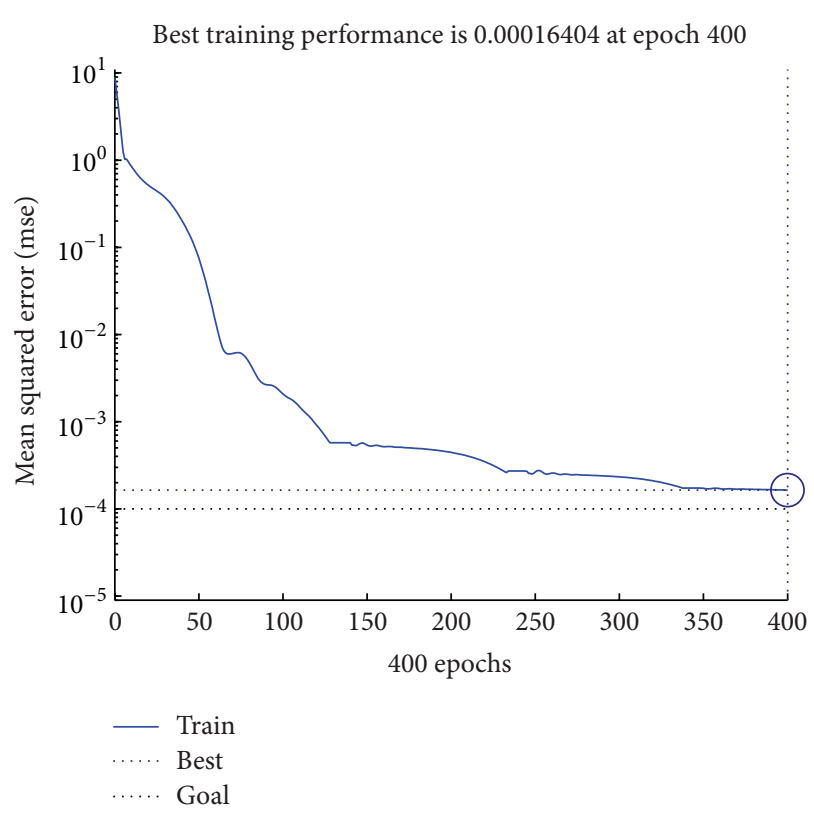

FIGURE 11: Dropping of deviation by training.

(96\%). The result shows that the fractal characteristics of partial discharge signals have a strong ability to describe pattern and the recognizer is effective. 
TABLE 11: Results of multi-SVM.

\begin{tabular}{lcccc}
\hline & & \multicolumn{2}{c}{ Discharge type } & \\
& Creeping discharge & Floating electrode discharge & Internal air discharge & External corona discharge \\
\hline Test sample number & 100 & 100 & 100 & 100 \\
Correct results number & 91 & 92 & 96 & 91 \\
Accuracy & $91 \%$ & $92 \%$ & $96 \%$ & $91 \%$ \\
\hline
\end{tabular}

TABLE 12: Accuracy comparison.

\begin{tabular}{lcccc}
\hline \multirow{2}{*}{ Algorithm } & & \multicolumn{2}{c}{ Discharge type } \\
& Creeping discharge & Floating electrode discharge & Internal air discharge & External corona discharge \\
\hline BP neural networks & $78 \%$ & $76 \%$ & $80 \%$ & $75 \%$ \\
ELM & $90 \%$ & $88 \%$ & $91 \%$ & $86 \%$ \\
Multi-SVM & $91 \%$ & $92 \%$ & $96 \%$ & $91 \%$ \\
\hline
\end{tabular}

\section{Conclusion}

Power cable is a very important part of the modern power system. Monitoring of power cables is directly related to the safety and stability of power system. So, it is necessary to build an accurate recognizer to recognize the discharge types correctly. The power cable model based on FDTD simulation is built to get the typical discharge signals as training samples, and fractal characteristics of the training samples are extracted and inputted into the recognizer. To make the results more accurate, multi-SVM recognizer is proposed in this paper. Finally, the BP neural networks recognizer and ELM recognizer are compared with multi-SVM recognizer proposed in this paper. The accuracy comparison shows that multi-SVM recognizer performs best (accuracy of each type over $90 \%$ ), especially the recognition of internal air discharge (96\%). The result shows that the fractal characteristics of partial discharge signals have a strong ability to describe pattern and the multi-SVM recognizer is effective.

\section{Conflict of Interests}

The authors declare that there is no conflict of interests regarding the republication of this paper.

\section{References}

[1] D. Wang, The study of transmission characteristics of the particle discharge signals based on three phase cross-bonded cables [Ph.D. thesis], North China Electric Power University, Beijing, China, 2008.

[2] K. Takeuchi, N. Amemiya, T. Nakamura, O. Maruyama, and T. Ohkuma, "Model for electromagnetic field analysis of superconducting power transmission cable comprising spiraled coated conductors," Superconductor Science and Technology, vol. 24, no. 8, Article ID 085014, 2011.

[3] R. Ambikairajah, B. T. Phung, J. Ravishankar, T. R. Blackburn, and Z. Liu, "Detection of partial discharge signals in high voltage XLPE cables using time domain features," in Proceedings of the 30th Electrical Insulation Conference (EIC '11), vol. 47, pp. 364-367, June 2011.

[4] A. Beroual and V.-H. Dang, "Fractal analysis of lightning impulse surface discharges propagating over pressboard immersed in mineral and vegetable oils," IEEE Transactions on Dielectrics and Electrical Insulation, vol. 20, no. 4, pp. 1402-1408, 2013.

[5] X. Chen, Y. Cheng, Z. Zhu, B. Yue, and X. Xie, "Insulating fault diagnosis of XLPE power cables using multi-parameter based on artificial neural networks," in Advances in Neural NetworkISNN 2005, vol. 3498 of Lecture Notes in Computer Science, pp. 609-615, Springer, Berlin, Germany, 2005.

[6] M. Wang and X. W. Li, "Power cable fault recognition using the improved PSO-SVM algorithm," Applied Mechanics \& Materials, vol. 427-429, pp. 830-833, 2013.

[7] L. Nanni, A. Lumini, D. Gupta, and A. Garg, "Identifying bacterial virulent proteins by fusing a set of classifiers based on variants of Chou's Pseudo amino acid composition and on evolutionary information," IEEE/ACM Transactions on Computational Biology and Bioinformatics, vol. 9, no. 2, pp. 467-475, 2012.

[8] B.-J. M. Webb-Robertson, W. R. Cannon, C. S. Oehmen et al., "A support vector machine model for the prediction of proteotypic peptides for accurate mass and time proteomics," Bioinformatics, vol. 24, no. 13, pp. 1503-1509, 2008.

[9] M. Johannes, J. C. Brase, H. Fröhlich et al., "Integration of pathway knowledge into a reweighted recursive feature elimination approach for risk stratification of cancer patients," Bioinformatics, vol. 26, no. 17, pp. 2136-2144, 2010.

[10] H.-J. Dai, P.-T. Lai, and R. T.-H. Tsai, "Multistage gene normalization and SVM-based ranking for protein interactor extraction in full-text articles," IEEE/ACM Transactions on Computational Biology and Bioinformatics, vol. 7, no. 3, pp. 412420, 2010.

[11] X. Xu, D. Niu, P. Wang, Y. Lu, and H. Xia, "The weighted support vector machine based on hybrid swarm intelligence optimization for icing prediction of transmission line," Mathematical Problems in Engineering, vol. 2015, Article ID 798325, 9 pages, 2015.

[12] J. A. Hunter, P. L. Lewin, L. Hao, C. Walton, and M. Michel, "Autonomous classification of PD sources within three-phase 11 kV PILC cables," IEEE Transactions on Dielectrics and Electrical Insulation, vol. 20, no. 6, pp. 2117-2124, 2013.

[13] W. Gang and T. Ju, "Study of minimum box-Counting method for image fractal dimension estimation," in Proceedings of the China International Conference on Electricity Distribution (CICED '08), pp. 1-5, December 2008. 
[14] H.-C. Chen, "Fractal features-based partial discharge pattern recognition using extension method," in Proceedings of the 4th International Conference on Intelligent Systems Design and Engineering Applications, pp. 274-277, IEEE, January 2012.

[15] L. Qi and X. Cui, "Electromagnetic transient analysis of cable system based on FDTD method," Acta Electronica Sinica, vol. 34, pp. 525-529, 2006.

[16] R. Papazyan, P. Pettersson, and D. Pommerenke, "Wave propagation on power cables with special regard to metallic screen design," IEEE Transactions on Dielectrics and Electrical Insulation, vol. 14, no. 2, pp. 409-416, 2007.

[17] M. Wang, P. Wang, J.-S. Lin, X. Li, and X. Qin, "Nonlinear inertia classification model and application," Mathematical Problems in Engineering, vol. 2014, Article ID 987686, 9 pages, 2014.

[18] R. V. Maheswari, P. Subburaj, B. Vigneshwaran, and L. Kalaivani, "Non linear support vector machine based partial discharge patterns recognition using fractal features," Journal of Intelligent \& Fuzzy Systems, vol. 27, no. 5, pp. 2649-2664, 2014.

[19] B. L. Liu, "Study on the fault diagnosis of turbine based on support vector machine," Applied Mechanics and Materials, vol. 55-57, pp. 1803-1806, 2011.

[20] J. Cao, S. Sun, and X. Duan, "A multi-classification algorithm based on support vectors," in Proceedings of the IEEE $3 \mathrm{rd}$ International Conference on Information Science and Technology (ICIST '13), pp. 305-307, IEEE, March 2013.

[21] C. Q. Men and W. J. Wang, "A randomized ELM speedup algorithm," Neurocomputing, vol. 159, pp. 78-83, 2015.

[22] R. Ahila, V. Sadasivam, and K. Manimala, "An integrated PSO for parameter determination and feature selection of ELM and its application in classification of power system disturbances," Applied Soft Computing, vol. 32, pp. 23-37, 2015.

[23] H. Chen, J. Peng, Y. Zhou, L. Li, and Z. Pan, "Extreme learning machine for ranking: generalization analysis and applications," Neural Networks, vol. 53, pp. 119-126, 2014. 

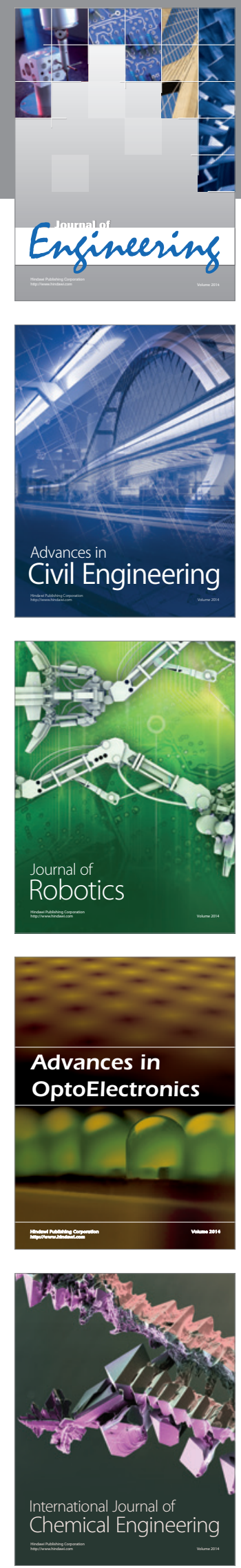

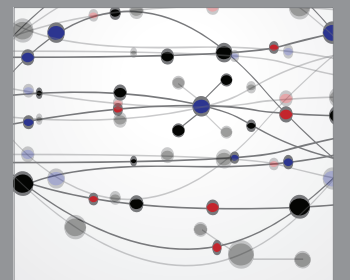

The Scientific World Journal
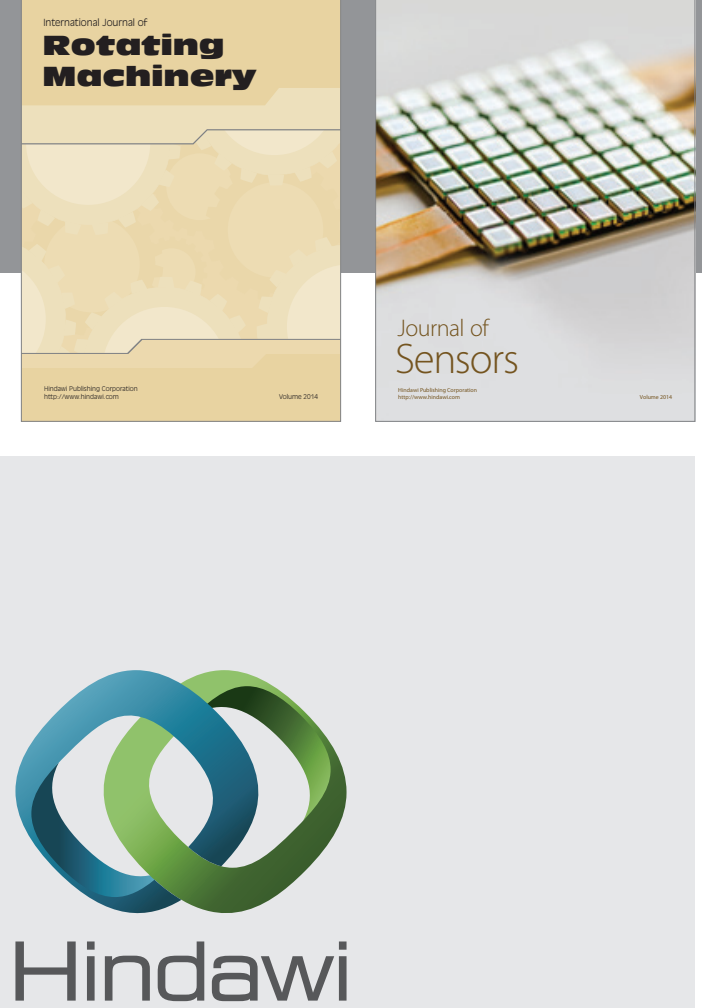

Submit your manuscripts at http://www.hindawi.com
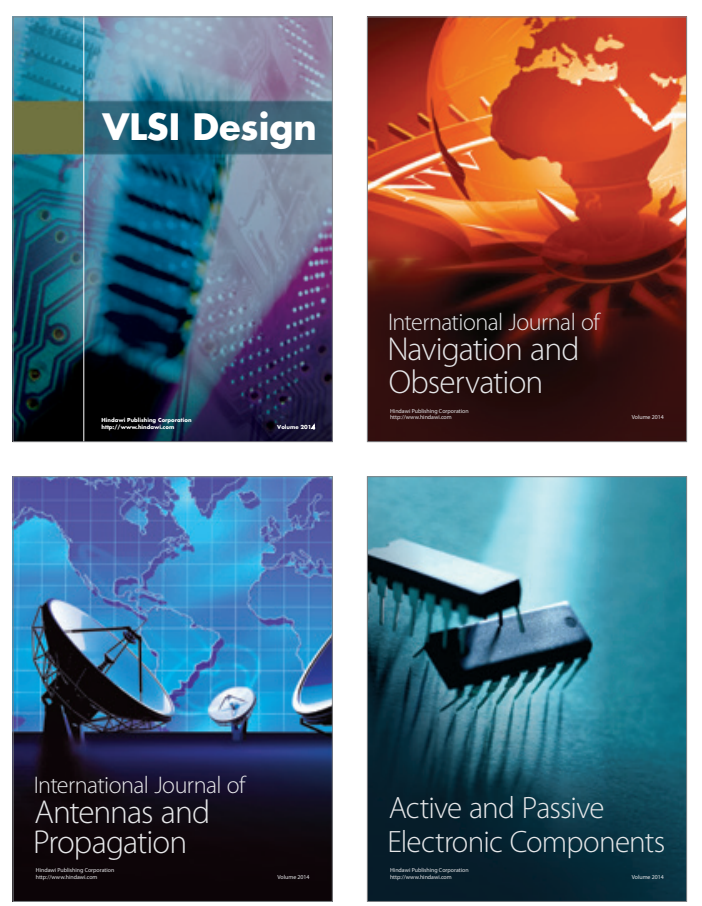
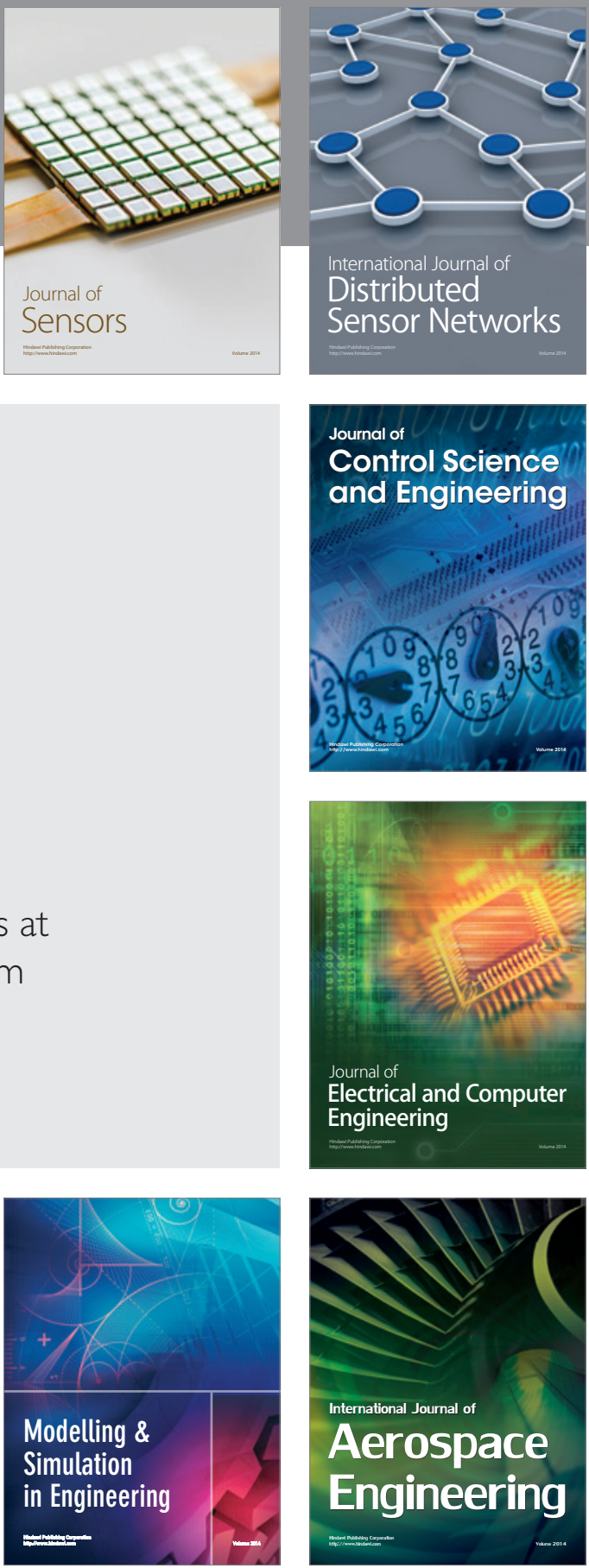

Journal of

Control Science

and Engineering
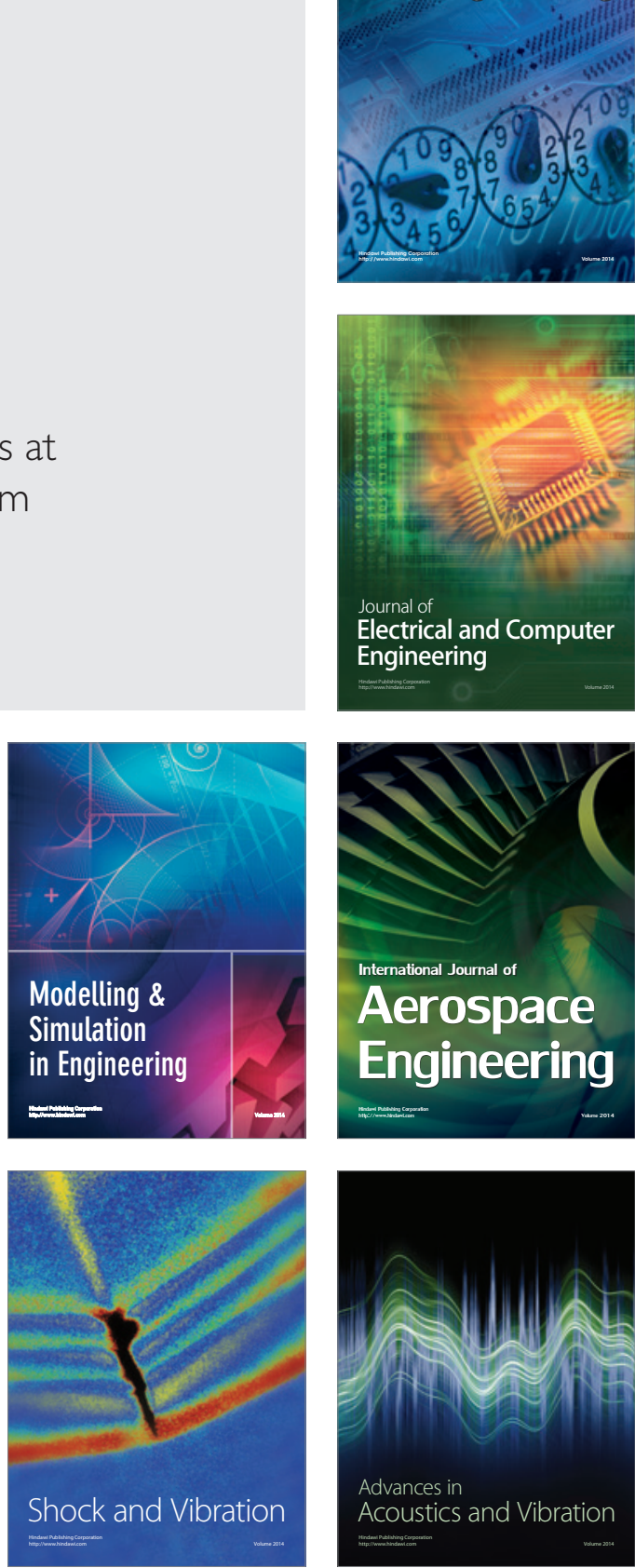